\section{CITATION INDEXING}

\section{Science Citation Index 1964 Annual Cumulation}

An International Interdisciplinary Index to the Literature of Scionce and Technology in Eight Parts. Part 1: Citation Index, A-Duke, Cols. 1-3168, Part 2: Citation Index, Duke-Kofe, Cols. 3169-6584, Part 3: Citation Index, Koff-Roes, Cols. 6585-10000, Part 4: Citation Index, Roes-Zzeb and Anonymous, Cols. 10001-13518, Part 5: Citation Index, Patent Citation Index and Acknowledgements, Cols. 13519-16404, Part 6: Source Index, Anonymous and A-Geye, Cols. 1-2658, Part 7: Source Index, Geye-Ogre, Cols. 2659-5316, Part 8: Source Index, Ogre-Zz, Cols. 5317-7893. Editor: E. Garfield. (Philadelphia: Institute for Scientific Information, Inc., 1965.) 1,250 dollars.

THE Science Citation Index for 1961 was reviewed in Nature, 203, 446 (1964), when an account was given of the technique for using a citation index. The Index for 1964 is an improvemont in many ways. It was published on a quarterly basis during the year, and the volumos reviewed hero represent the annual cumulation. Whereas the Index for 1961 appeared as the result of a rosearch grant from the National Science Foundation and the National Institutes of Health, the present index can be considered as the first to be issued on a production basis, and it is obviously an advantage that the publication delay has beon reduced.

The other major change has been the inclusion of a list of all the citing papers. This greatly improves the convenience of using the Index, since, as is inevitable in this type of index, many of the citing papers will prove to be quite irrelevant for the subject of the search. With the earlier Index, this point could only be established by checking the actual journal; now, by referring to the list of citing papers, from the titles one can correctly reject many of the non-relevant papers. The physical price to be paid for this is that the number of volumes has increased from five to eight; the financial price is that the cost has risen to 1,250 dollars. Against this, there has been an incressed coverage; the source journals have increased only to 700 from 613 , but the separate journal papers have risen from 102,000 to 151,000 . In addition, for the first time, patents are included, and the 47,000 patents issued in the United States in 1964 bring the total of source items up to 199,000 . It is a disappointment to find that the standard computer print-face, severely reduced in size, is still being used, and also that the Index is still heavily biased towards the lifo sciences. It is to be hoped that the balance will be redressed in the Index for 1965 , when, it is stated, more than 1,000 journals will be covered.

The present value and future potentialities of the Science Citation Index are difficult to assess. The cost is such that it can be considered prohibitive for individuals, yot for a library it would represent only a fraction of the cost of one human indexer. The usefulness of the Index would bo greater if its coverage were wider, yet this would result in an increase in cost unless there was a corresponding increase in the number of purchasers. Before placing a subscription, a librarian needs to be reasonably satisfied that the citation index is not only a useful tool, but also that it will not cease publication within a year or so, for it is only when the library shelves have a run covering at least five yoars that the majority of users of the library will be prepared to accept it as a method of retrieving information. Only then will it bo possible to assess how usefully it can fulfil its basic purpose and find whother its obvious technical weaknesses are obviated by the flexibility and ease of compilation. The use of a computer for this purpose makes possible a number of interesting alternatives. Already an Automatic Subject Citation Alert (ASCA) service is being offered, by which subscribers can obtain regularly citations to papers by selected authors. There is no reason why cumulations covering selected subject fields should not be issued at a price which would appear more reasonable to prospective purchasers. There are also, of course, known ways of improving the operational performance of a citation index; some of these, by increasing the complexity, would raise the cost, but improvement in the citing habits of authors would do more than anything else to increase the usefulness of a citation index. Perhaps the time is coming when editors of sciontific journals should take the samo activo steps to obtain a high quality of citing as many have recently been doing for abstracts and titles. C. W. CLEVERDON

\section{COMMUNICATION, THE SOCIAL WEB}

\section{Communication Processes}

Edited by Frank A. Geldard. (NATO Conference Series, Vol. 4.) Pp. $x+299$. (London and New York: Pergamon Press, 1965.) 100s. net.

COMMUNICATION Processes is Volume 4 of the

NATO Conforence Series of volumes, being the Proceedings of a symposium held in Washington in 1963 , under the sponsorship of the NATO Advisory Group on Human Factors. This military sponsorship should not mislead one, however, because the contents will be of interest to a wide civil readership and, indeed, have, on the whole, no limitation to military situations at all, but relate to many kinds of formal social institution.

The book is divided into five principal sections: (1) data presentation; (2) data transmission; (3) language barriers and language training; (4) group communication; (5) man-computer communication. Each consists of an introductory paper with very extensive attendant discussions. About one-fifth of the whole contents is written in French. The conference was concerned essentially with human factors of communication and not at all with electronics or engineering; of course, human factors are themselves very important to engineers. The participants may be regarded as being the most prominent psychologists and sociologists concerned with communication processes-an impressive galaxy of stars from the various NATO countries, representing a wide rango of interests and opinions.

At least a third of this book is devoted to 'pragmatic' aspects of communication; that is, to the importance of personal prejudices, to people's positions in a social hiorarchy, to their cultural backgrounds and to noncognitive factors of language; the subtle connotations of language, one's image of oneself and other determinants of human communication. This aspect of the book is valuable, for such vital matters are not always evident to those engaged in the development of technical means of communication, and they are ignored by those who take a strictly logical view of semantics, a viow which seems so prevalent to-day. A 'yes' can be turned to a 'no' by a smile, or by one's knowledge of the speaker's reputation. Meanings are not properties of words but are relationships, between words and people, in various situations and conditions. These pragmatic factors can be of particular importance in cross-cultural communication, already hampered by the strict untranslatability of languages. However, John Black, of Ohio State University, plays down these factors, in his paper "Language Barriers and Language 'Training", and quotes several historical examples of people, drawn from different cultures, nevertheless getting on together quite well; Columbus, for example, among the natives, and Marco Polo. But his examples are very special ones and, besides, the fact that such people were not promptly murdered does not mean that they were understood correctly. I cannot help quoting the old story of Captain Cook who, when he asked a nativo "What's that silly-looking animal called ?", was given the answer: "Kangaroo", which means in Aborigine "I don't know" (so I am told !). Of course, the "word 